\title{
Effect of ruthenium oxide content on the electrochemical characteristics of a supercapacitor with a carbon black electrode formed by electrophoretic deposition
}

Kakovkina Ju.I. ${ }^{1,2}$, Lebedev E.A. ${ }^{1}$, Kuzmin D.A. ${ }^{1}$, Kitsyuk E.P. ${ }^{2}$, Alekseyev. A. V ${ }^{1}$, Ryazanov R.M. $^{2}$

1- National Research University of Electronic Technology - MIET

2- SMC "Technological Centre"

Abstract: Controlling the ratio of capacitance and power of supercapacitors by changing the composition of the electrodes will allow to create optimal power systems for specific applications. For the formation of such electrodes, a method is required that combines the possibilities of creating a multicomponent composite with a high degree of uniformity of composition and morphology over the layer thickness. An example of such a method can be the eco-friendly method of electrophoretic deposition used in this work, which makes it possible to locally deposit a composite material from multicomponent suspensions at room temperature. We present an approach related to electrophoretic deposition from a suspension of composite material SuperC- $\mathrm{RuO}_{2}$, in which the ratio of the components can be changed to vary the proportion of electrochemical and electrical double layer storage. Nanocarbon, which has a large surface area, and ruthenium oxide with a significant electrochemical capacity, in combination, will allow combining high power and capacity in one device, and their ratio will determine the proportion of electrochemical and electrical double layer storage. In this work, approaches are investigated and recommendations are given for increasing the stability of suspensions, the effect of the composition of the suspension on the composition of composite electrodes and their capacitive and power characteristics is determined.

Keywords: supercapacitor; electrophoretic deposition; nanocarbon; energy storage

\section{Introduction}

The capacity and power of supercapacitors are generally determined by the surface area on which the charge accumulates. Traditional electric double-layer capacitors (EDLC), which accumulate charges electrostatically at the electrode / electrolyte interface (non-Faradaic process), demonstrate greater power when compared to other devices, such as batteries. However, for power up to $15 \mathrm{~kW} / \mathrm{kg}$, their capacity is only about 5-10 Wh / kg [1]. 
A further development of such structures was supercapacitors using fast and reversible electrochemical reactions on the surface of electrode materials during intercalation or adsorption of ions from the electrolyte (pseudo capacitors), where the accumulated charge is neither purely capacitive nor volume Faraday. Accordingly, the prefix "pseudo" with "capacitor" is used to show the features of a capacitor in its electrochemical signature (CV and CDs) and to differentiate them from EDLC in regards to the charge storage mechanism [2]. These devices can already demonstrate power and capacity in the order of $16.1 \mathrm{~kW} / \mathrm{kg}$ and $24.9 \mathrm{Wh} / \mathrm{kg}$, respectively [3]. At the same time, the kinetics of the ongoing redox reactions is very fast. Its speed is not limited by the diffusion of ions through the electrode material, which fundamentally distinguishes the characteristics of pseudocapacitors from batteries.

The electrodes in pseudocapacitors are oxides and hydroxides of transition metals. The main requirements for them are high theoretical specific capacity, electrical conductivity, and stability during cycling, as well as availability and low toxicity. Among metal oxides, ruthenium oxide $\left(\mathrm{RuO}_{2}\right)$ is a typical material for pseudocapacitors due to its remarkably high theoretical specific capacitance (1358 $\mathrm{F} \mathrm{g}^{-1}$ ) and exceptional properties such as metallic-type conductivity, wide potential window, and highly reversible redox reaction [4]. $\mathrm{RuO}_{2}$ has a high specific capacitance, several problems have been observed such as short life time and weak performance at high charge/discharge rates [5-7]. The volume of $\mathrm{RuO}_{2}$ changes by the redox and oxidation reaction of $2.54 \AA$ to $3.02 \AA$, resulting deterioration under repeated cycles. The short life of the $\mathrm{RuO}_{2}$-based supercapacitor is lead to limits the practical applications of $\mathrm{RuO}_{2}$ as supercapacitor electrodes [8]. A method to improve the cycling stability of hydrous $\mathrm{RuO}_{2}$ and obtain preferable electrochemical performance may be the use of hybrid technology by adding various carbon based materials (activated carbon, carbon nanotubes, and graphene) to $\mathrm{RuO}_{2}$ nanoparticles [9-13].

In this case, carbon materials provide mechanical strength and electrical contact between all particles that can be disturbed due to changes in the volume of materials during charging and discharging. Also nanomaterials have been very attractive in the research of electrochemical capacitors, due to their short ion diffusion length and high surface to volume ratio. In this application, many different 1,2,3-dimensional modifications of the latter have been studied, such as carbon nanotubes (CNT), graphene and reduced graphene oxide (ROG), carbon nanofibers (CNF), graphene nanofoams (GNF), etc. [4]. In this work, nanoparticles (Super C45) were used. 
To form the composite material of the electrodes, the method of electrophoretic deposition (EFO) was used, which has a number of advantages over other methods: direct and screen printing[14], solvothermal synthesis [15], sol-gel [16], layer-by-layer deposition [17], aerosol spraying [18], chemical deposition from the gas phase [19]. EFO allows to control over a wide range of the ratio of components, coating thickness, density and porosity [20], in contrast to direct printing and sol-gel techniques. Also, the advantages of the method are its low temperature and selectivity, its suitability for local application of functional material to current collectors with a predetermined shape and topology. The composite material can be applied in one technological operation with high adhesion to the substrate or the layers covering the substrate. In this case, it is optional to use a binder that can increase the electrical resistance of the electrode and decrease the active surface area of the metal oxide. In addition, the binder interface is often damaged by the repeated charge/discharge process due to the volumetric expansion/contraction of $\mathrm{RuO}_{2}[21]$.

In this work, we study the features of obtaining high-capacity electrodes of supercapacitors by electrophoresis from suspensions with different compositions, as well as the influence of deposition parameters on the electrophysical characteristics of the structures formed. Commercially available carbon nanoparticles (Super C45) with an average size of $100 \mathrm{~nm}$ and ruthenium hydroxide particles with a size of $30 \mathrm{~nm}$ are selected as the main materials to form the composite materials of the electrodes.

\section{Technology}

In this work, an approach to the creation of supercapacitors based on composite electrode materials will be considered. Ruthenium oxide (concentration range $0.05-0.3 \mathrm{mg} / \mathrm{ml}$ ) and Super C45 (fixed concentration $0.2 \mathrm{mg} / \mathrm{ml}$ ) have been proposed as the main materials. Nickel foil was used as a substrate. The solvent was a 1: 1 isopropyl alcohol + acetone combination.

The suspension was prepared in a cleaned $50 \mathrm{ml}$ test tube. Initially, a combination of components of different concentrations was added to the test tube. Then $10 \mathrm{ml}$ of solvent (a mixture of acetone and isopropyl alcohol in a 1: 1 ratio) was poured into the test tube. Dispersion of the resulting solution was carried out using a submersible disperser UZTA-0.1 / 28-O "Alena" in several stages:

1) 2 minutes of work at $20 \%$ power;

2) 2 minutes at $50 \%$ power; 
3) 30 minutes at $100 \%$ power.

The tube temperature at all stages was stabilized by a water cooling system in the range of $15 \pm 3^{\circ} \mathrm{C}$. In the next step, $40 \mathrm{ml}$ of the same solvent was added to $10 \mathrm{ml}$ of the whipped suspension, after which dispersion was continued for 10 minutes.

Blanks with dimensions of 50x20 mm were cut out from nickel tape $0.05 \mathrm{~mm}$ thick and washed in a solution of acetone and isopropyl alcohol (acetone: isopropyl alcohol: $\mathrm{H} 2 \mathrm{O}=1$ : 1: 1, by volume) in an ultrasonic bath "Sapphire" for 10 minutes. After that, the workpieces were washed in deionized water and processing in ultrasonic bath once again in an aqueous solution of nitric acid $\left(\mathrm{HNO}_{3}: \mathrm{H}_{2} \mathrm{O}=1: 4\right.$, by volume). A similar procedure was further performed in deionized water. The final stage of washing the blanks was drying them in isopropyl alcohol vapor.

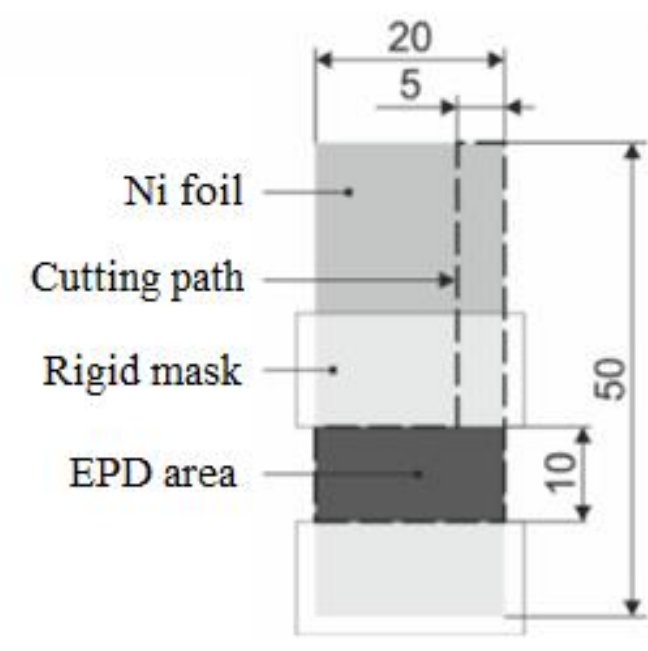

Figure 1 - Schematic representation and geometrical dimensions (in $\mathrm{mm}$ ) of a supercapacitor

The deposition was carried out in an electrophoretic cell, consisting of two electrodes - a cathode (nickel foil) and an anode (gold electrode), a container for a suspension, and a KEYSIGHT B2912A current source. To limit the deposition area $\left(1 \times 2 \mathrm{~cm}^{2}\right)$, the sample (cathode) surface was partially masked using a rigid mask made of sitall plates (Figure 1). The electric field strength varied from 50 to $150 \mathrm{~V} / \mathrm{cm}$, deposition was carried out in 1 cycle of $60 \mathrm{~s}$ or 5 cycles of $60 \mathrm{~s}$ each with suspension renewal. To determine the sediment mass, the samples were weighed before and after the sedimentation process on a Metler Toledo MT XP 205 balance. 
The particle size, surface morphology, and composition of the sediments were analyzed using a Jeol JSM-6010 PLUS / LA scanning electron microscope (SEM) with an energy dispersive X-ray spectroscopy (EDX) attachment.

The samples dried after electrophoretic deposition were prepared for a series of electrophysical measurements. A figure was cut out of foil with a formed composite coating, where a current collector leg with a width of about $5 \mathrm{~mm}$ fits to an area of $20 \times 10 \mathrm{~mm}^{2}$ with a functional material. At the same time, a vertical-mirror figure was made from pure nickel foil. They constitute the electrodes of the future electrochemical device. When functional areas of $20 \times 10 \mathrm{~mm}^{2}$ were superimposed on each other, a porous polypropylene membrane with a size of $30 \times 20 \mathrm{~mm}^{2}$ impregnated with $1 \mathrm{M} \mathrm{KOH}$ electrolyte was placed between them. The resulting sandwich structure was sealed with a laminator in a polypropylene film.

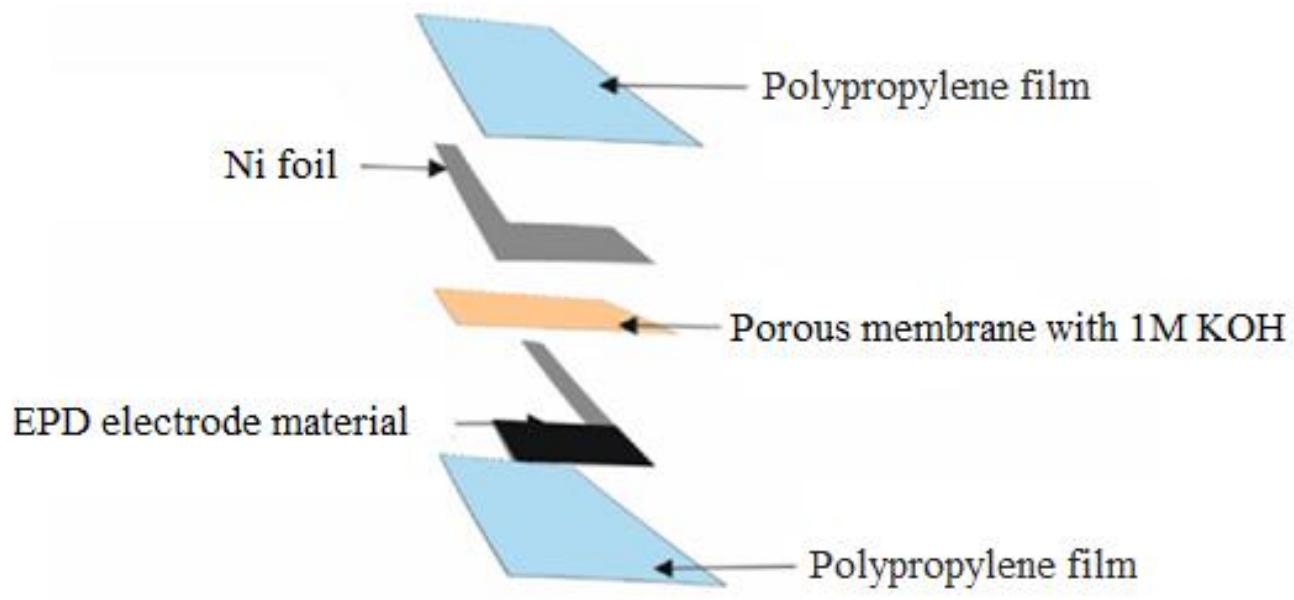

Figure 2 - Supercapacitor assembly diagram

In this form, the supercapacitor sample was kept for a day to impregnate the composite material with electrolyte. Immediately before measurements, the main part of the device was fixed in a clamp to maintain a constant distance between the plates. The negative electrode of the measuring device was connected to the plate with the applied functional material, and the positive electrode was connected to the clear foil.

Registration of cyclic sweeps and charge-discharge curves of prototypes of electrochemical energy storage devices was carried out using an Elins-45X potentiostat with the attached ES8 program for a PC. Cyclic sweeps were recorded at rates of 10 and $100 \mathrm{mV} / \mathrm{s}$ in the cell voltage range from 0 to $1000 \mathrm{mV}$. The charge-discharge was also carried out in the voltage range from 0 to $1000 \mathrm{mV}$ in the galvanostatic mode, while the current strength was selected in the range from 5 to $100 \mathrm{~mA}$ so that the discharge duration was at least 10 seconds. 


\section{Results}

\subsection{Deposition}

Suspension with the following composition: Super C45 (10mg) +cellulose $(10 \mathrm{mg})+\mathrm{RuO}_{2}$ (10 mg) + I (20 mg), +50 ml acetone/isopropanol (50:50) after some experiments was chosen for our process. The morphology and composition of the layers obtained from this suspension by the electrophoresis method were investigated by the SEM and EDX methods. The data obtained indicate a low density and a developed morphology of the layer with uniform deposition of all components contained in the suspension.

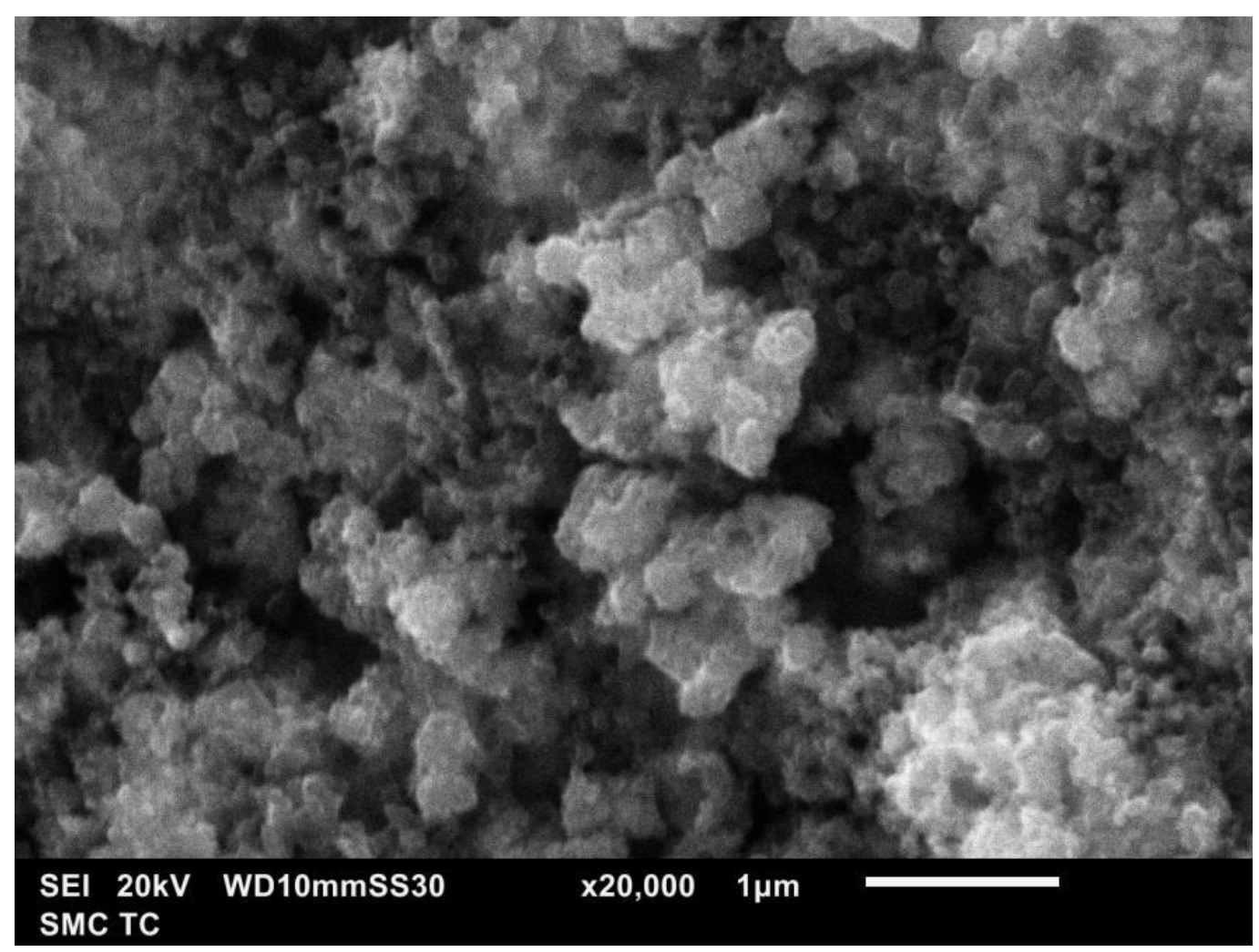

Figure 3-SEM of the electrode after 5 deposition cycles

SEM photos and EDX analysis of the electrophoretic-deposited electrode demonstrate uniform distribution of ruthenium oxide and carbon nanoparticles (Figures 3 and 4). 

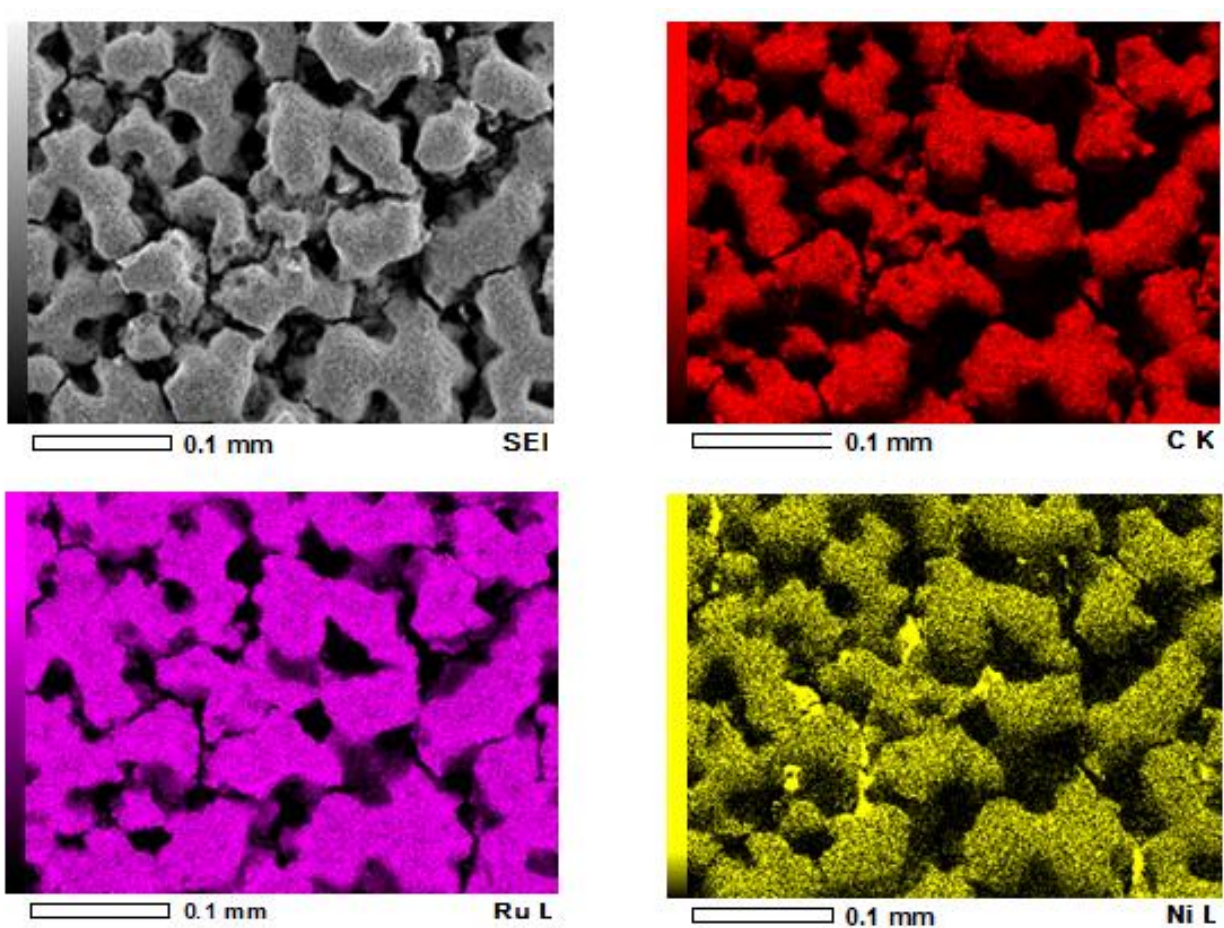

Figure 4-EDX surface mapping of the electrode by elements after 5 deposition cycles at $100 \mathrm{~V}$

Composition of this electrode according to EDC data is given in table 1.

Table 1. The quantitative content of elements in the composite electrode.

\begin{tabular}{|c|c|c|}
\hline Element & Mass \% & Atom \% \\
\hline $\mathrm{C}$ & 17,74 & 59,72 \\
\hline $\mathrm{O}$ & 3,38 & 8,55 \\
\hline $\mathrm{Ni}$ & 1,09 & 0,75 \\
\hline $\mathrm{Ru}$ & 76,20 & 30,47 \\
\hline $\mathrm{I}$ & 1,58 & 0,50 \\
\hline Total & 100 & 100 \\
\hline
\end{tabular}

Further, the dependences of the formation features of supercapacitor electrodes on the deposition process parameters were analyzed. It was found that the mass of the sediment grows almost linearly with increasing process voltage (figure 5). 


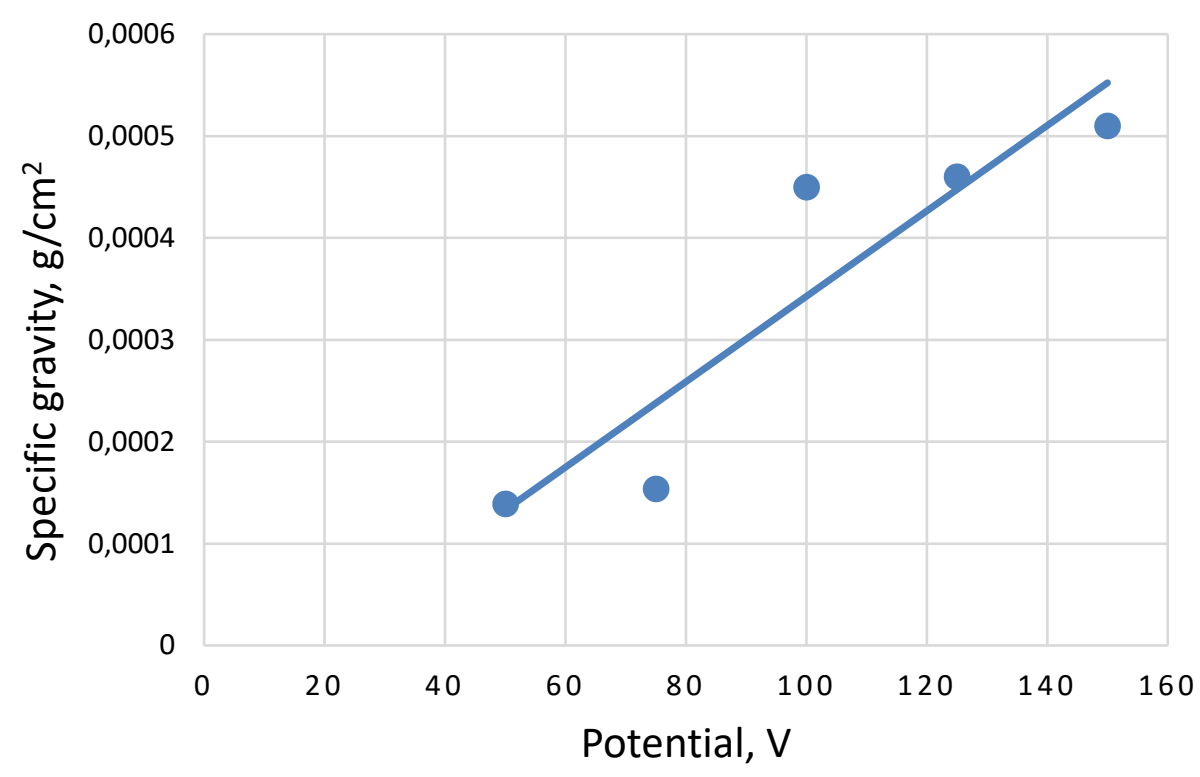

Figure 5 - Dependence of the specific gravity of the electrode on the electrophoresis voltage

At the same time, no clear dependence of the electrode mass on the amount of ruthenium oxide in the suspension was established (Figure 6).

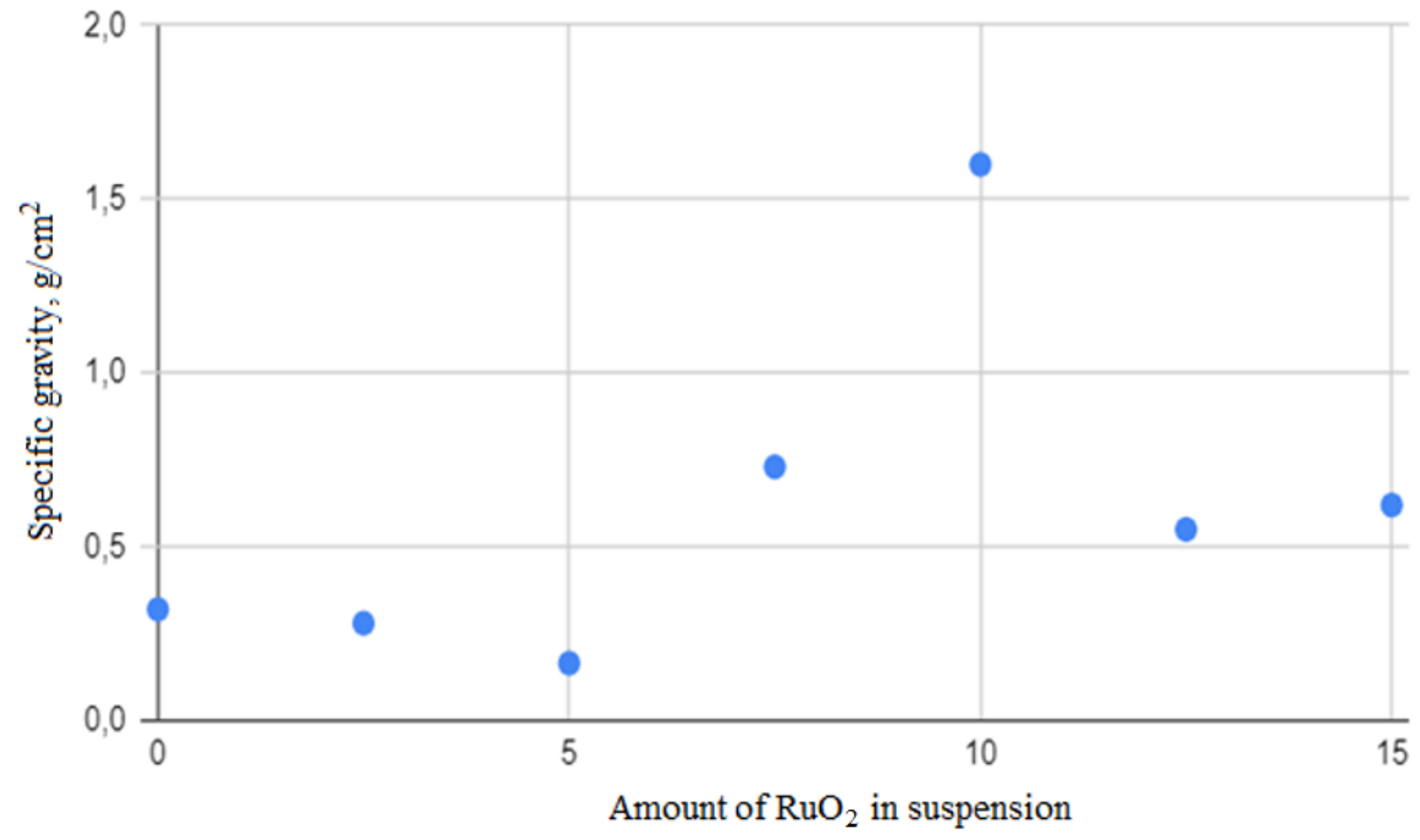

Figure 6 - Dependence of the specific gravity of the electrode on the $\mathrm{RuO}_{2}$ content in the suspension after 1 deposition cycle 
Also, the same distribution of the electrode is observed with an increase in the number of deposition cycles from 1 to 5 .

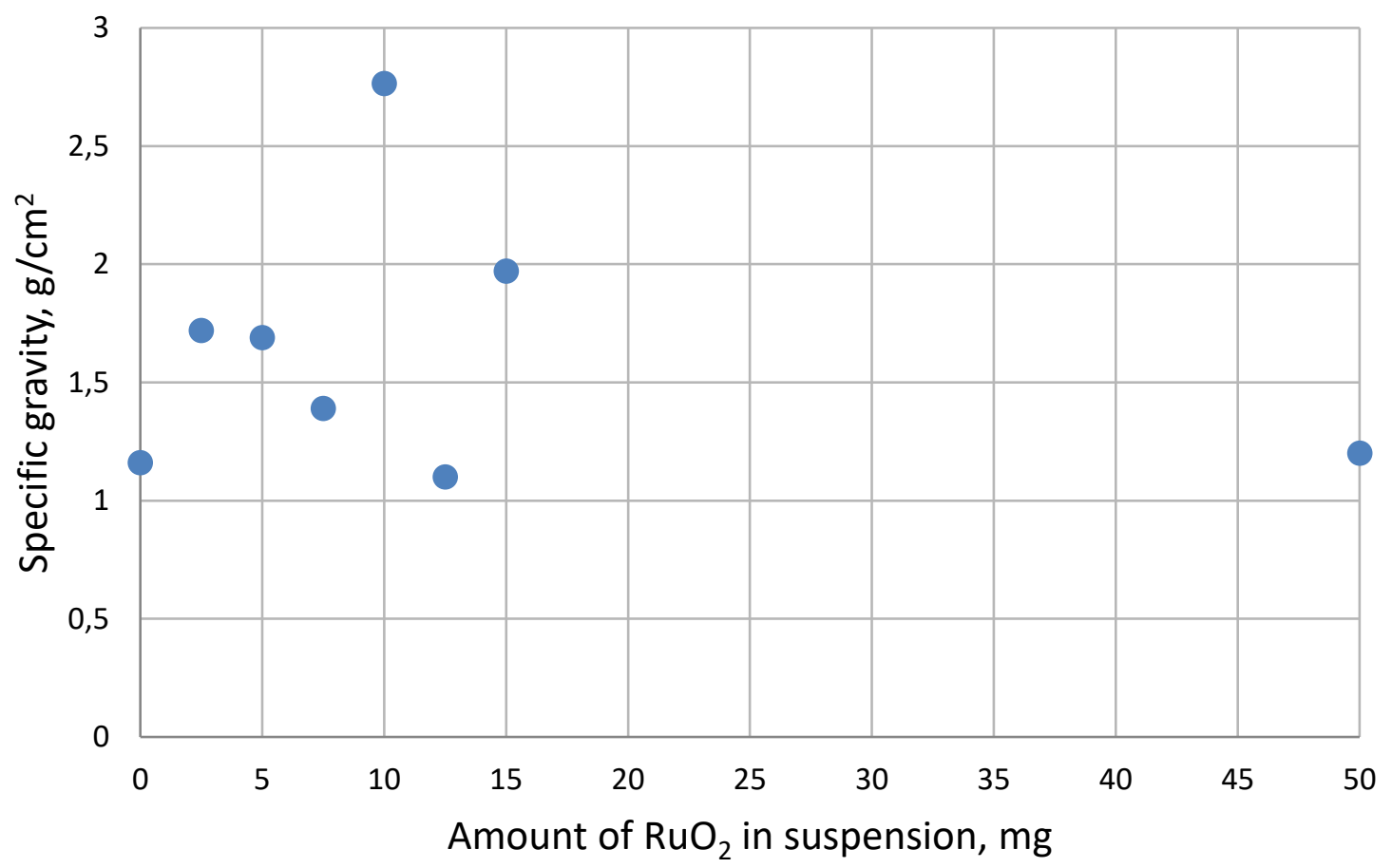

Figure 7 - Dependence of the specific gravity of the electrode on the $\mathrm{RuO}_{2}$ content in the suspension after 5 deposition cycles

Since the key role in this process is played not only by the composition of the suspension, but also by its stability, experiments were carried out on the influence of the holding time of the suspension on the results of electrophoretic deposition. The electrodes were formed from the slurry just made after 4 and 8 hours. As can be seen from the data in Figure 7, the amount of material deposited from the new suspension is slightly larger than from the aged. 


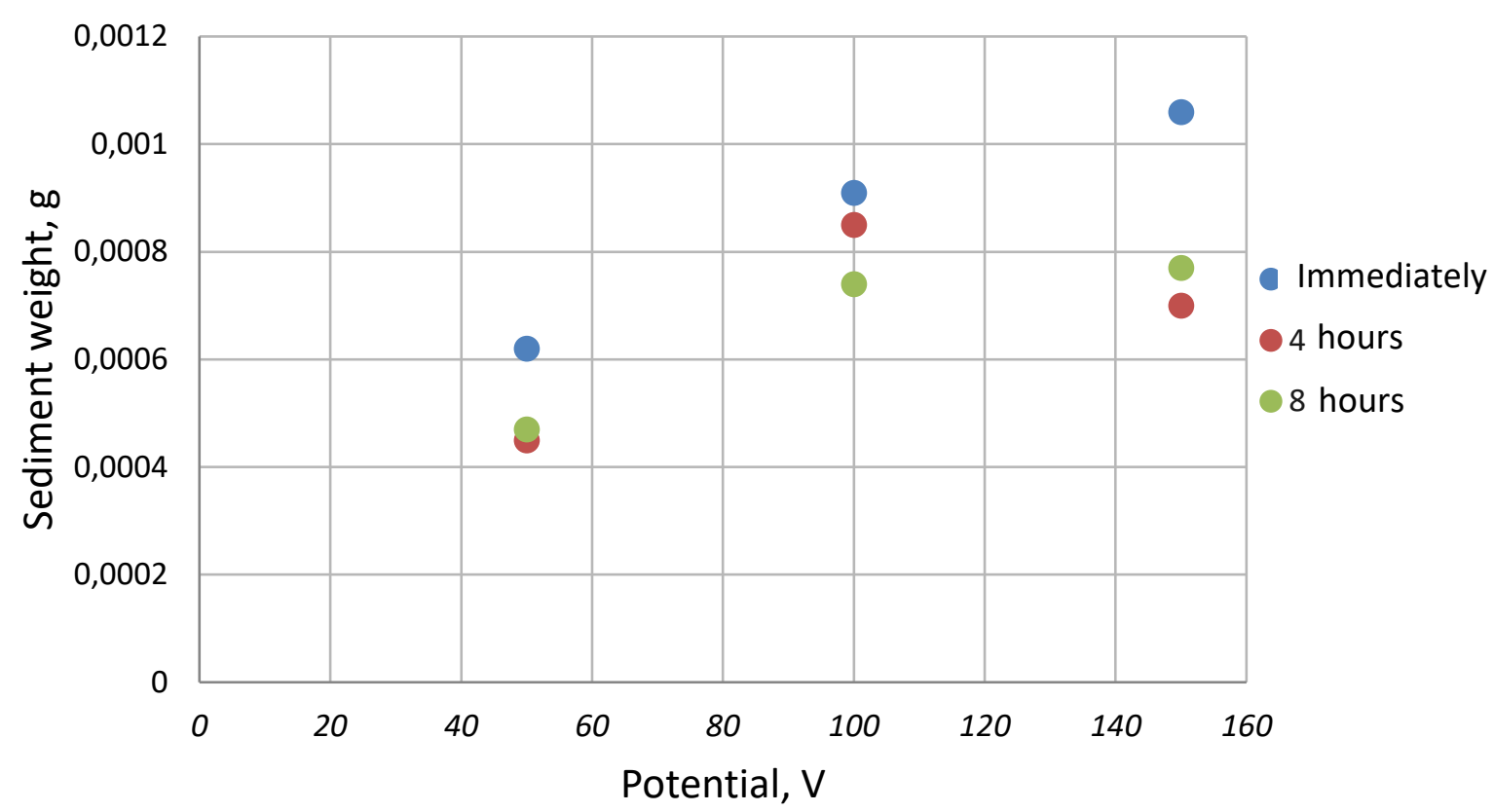

Figure 8 - Influence of the suspension aging time on the mass of the deposited electrode

Symmetric supercapacitors were made on the basis of these electrodes and their electrochemical characteristics were measured. Specific capacity of the samples with electrodes formed in 1 cycle and 5 cycles were investigated at a sweep rate of 10 and $100 \mathrm{mV} / \mathrm{s}$ (Figures 9 and 10).

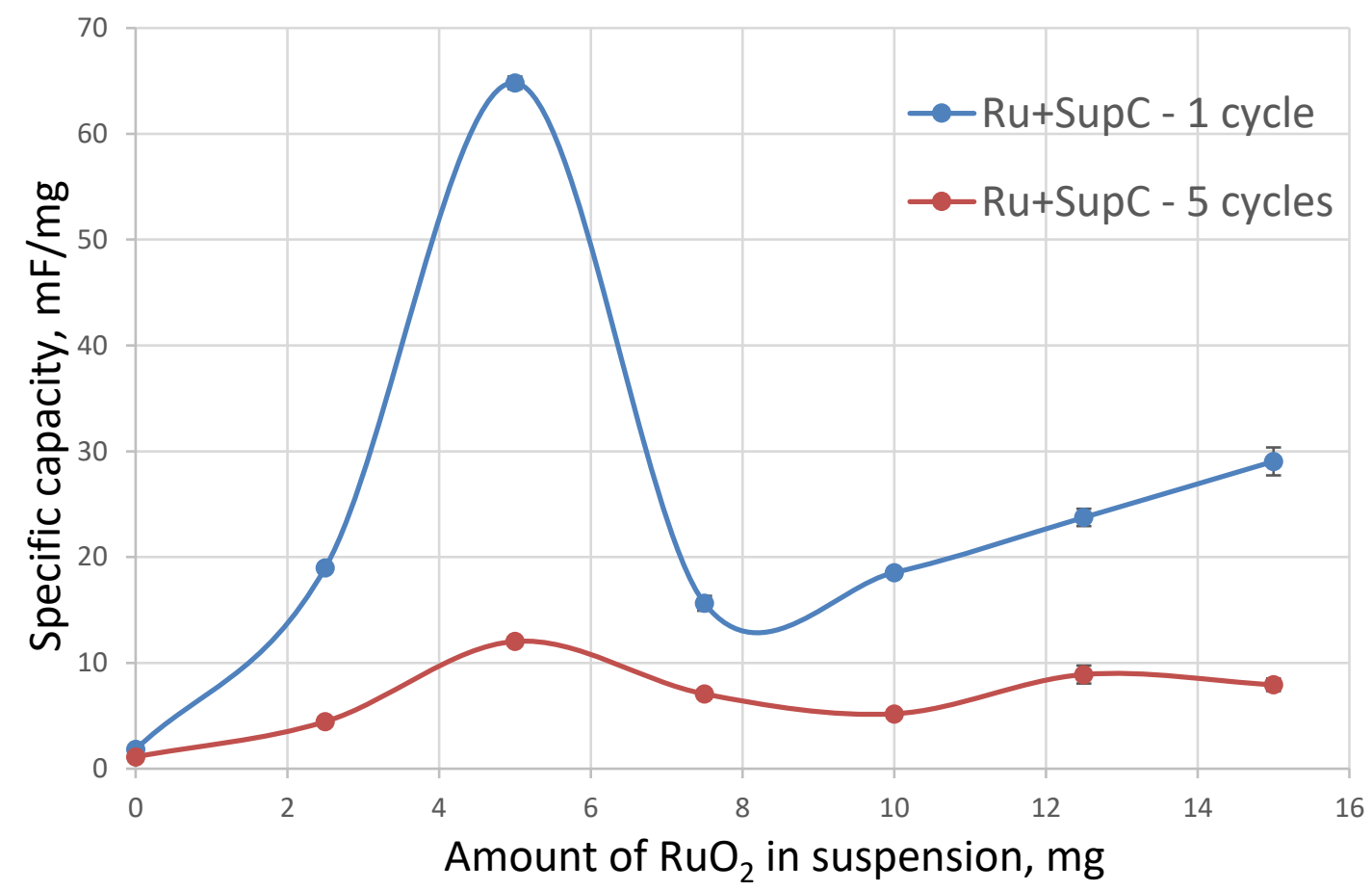

Figure 9 -Specific capacity of the formed supercapacitors with a sweep rate of $10 \mathrm{mV} / \mathrm{s}$ 


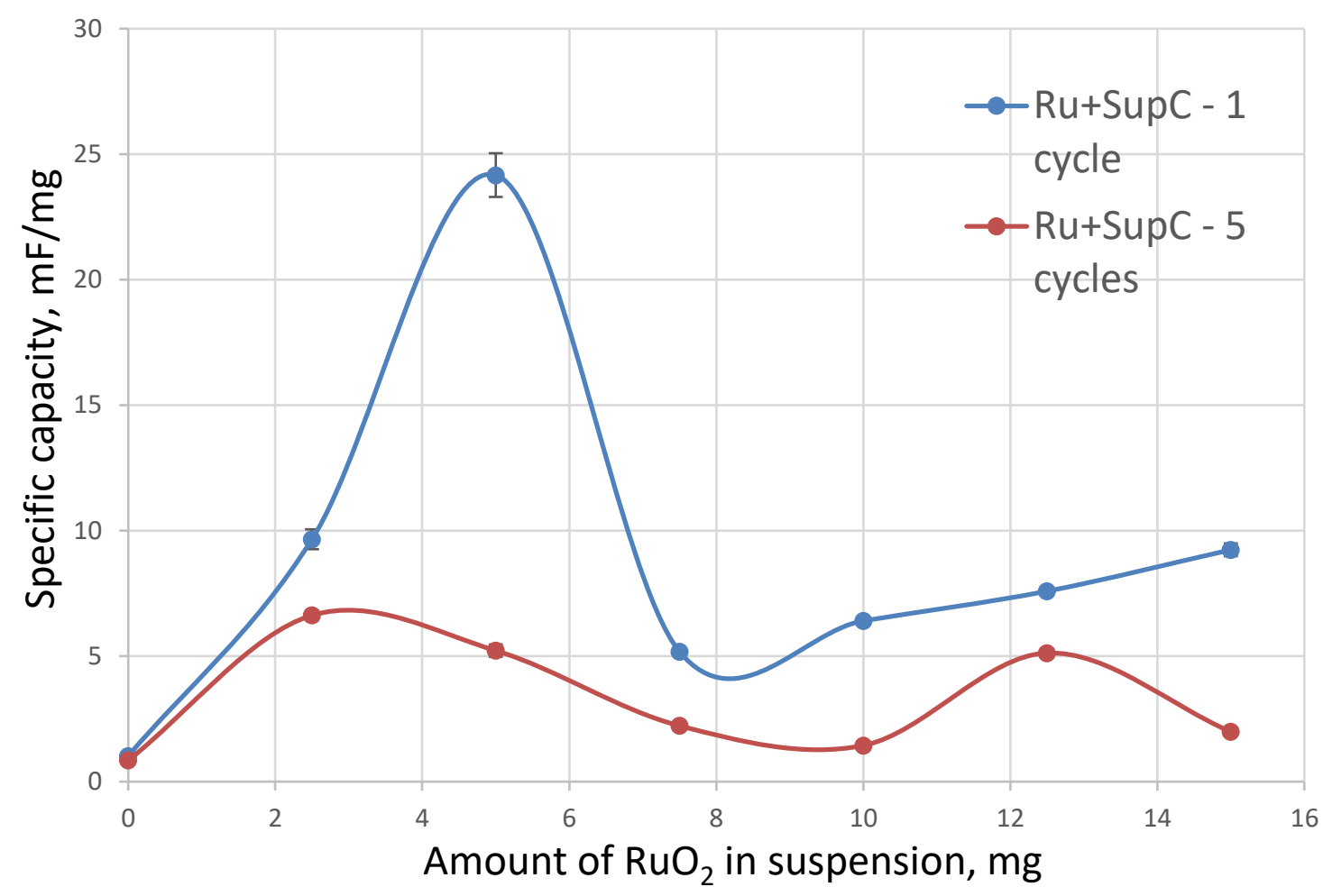

Figure 10 - Specific capacity of the formed supercapacitors with a sweep rate of $100 \mathrm{mV} / \mathrm{s}$

Voltammetry measurements of samples made from suspension with $5 \mathrm{mg} \mathrm{RuO}_{2}$ is shown at figures 11 and 12. Peaks associated with the occurrence of reversible electrochemical factors associated with ruthenium oxide can be identified on the curves.

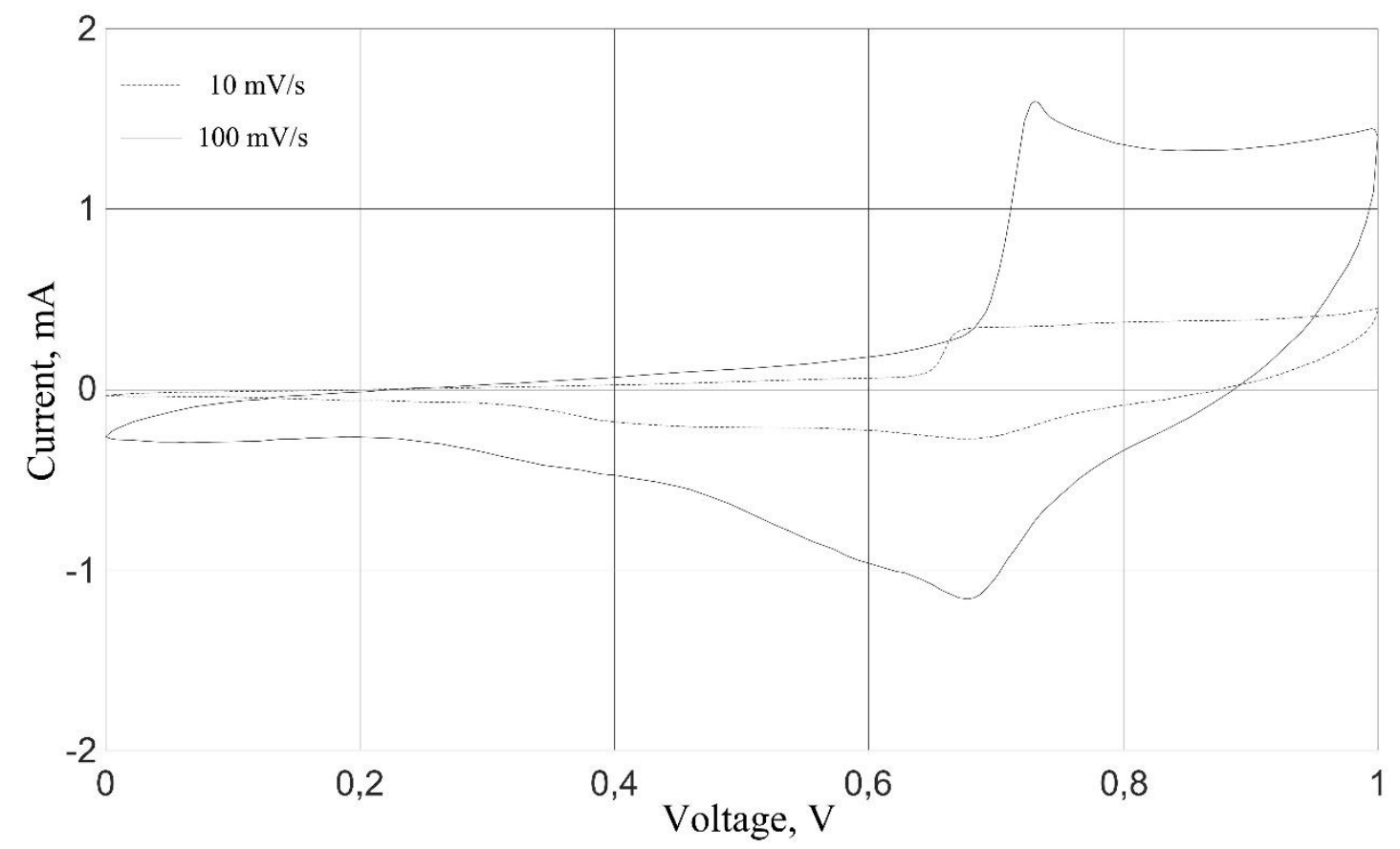

Figure 11. Cyclic sweep of the formed supercapacitor with $1 \mathrm{EPD}$ cycle at $10 \mathrm{mV} / \mathrm{s}$ and $100 \mathrm{mV} / \mathrm{s}$ 


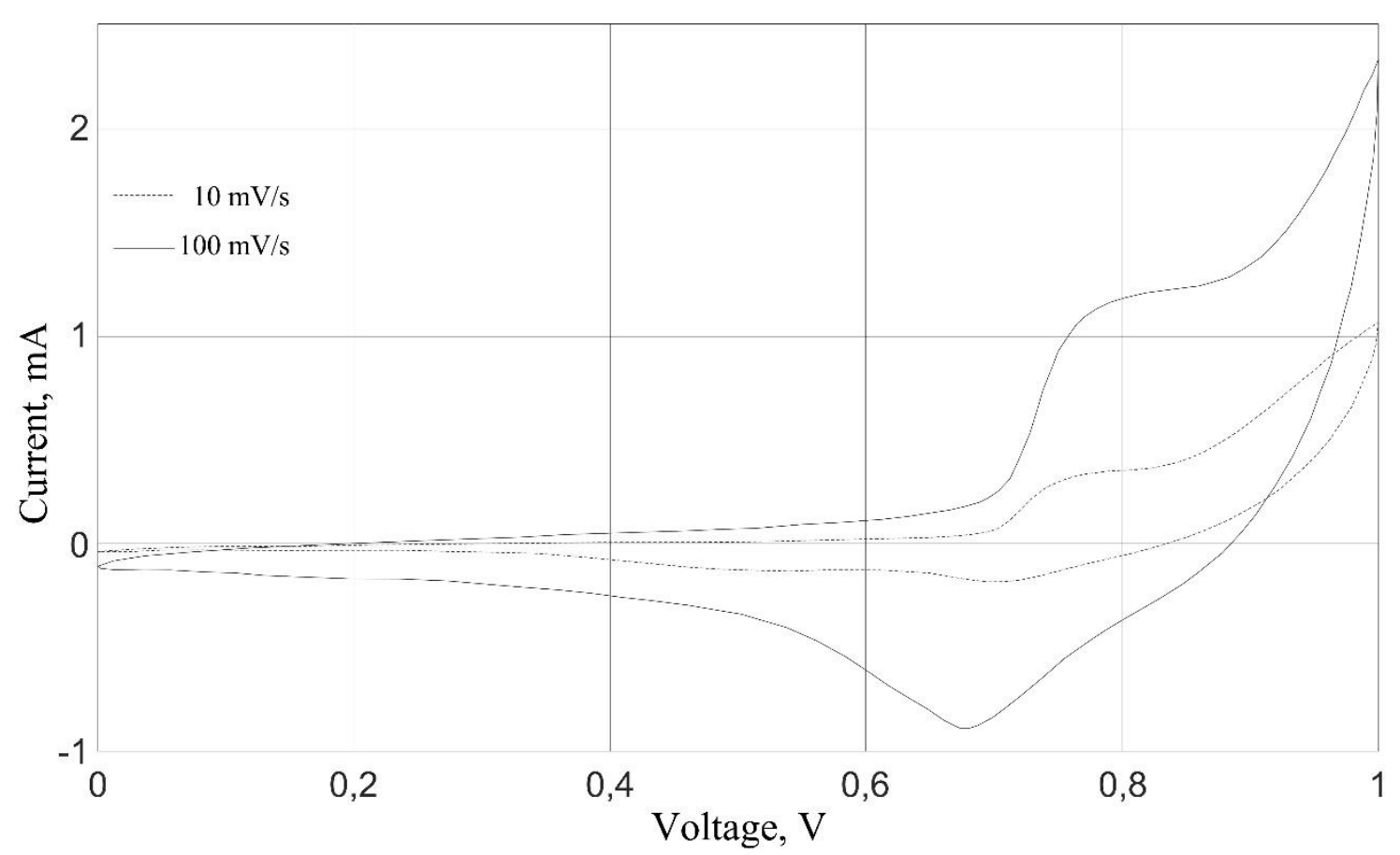

Figure 11. Cyclic sweep of the formed supercapacitor with 5 EPD cycle at $10 \mathrm{mV} / \mathrm{s}$ and $100 \mathrm{mV} / \mathrm{s}$

The contribution of electrochemical reactions to the total capacity of a supercapacitor is greater for a sample prepared in 1 cycle of electrophoretic deposition of an electrode material. This indicates the negative impact of excessive deposition processes that lead to isolation of ruthenium particles and a decrease in electrode capacity.

\section{Conclusions, Outlooks, and Opportunities for Future Development}

In this work we investigate some peculiarities of the electrophoretic deposition of composite electrode material based on $\mathrm{RuO}_{2}$-carbon nanoparticles for supercapacitors. it was defined that despite the fact that the dependence of the electrode mass on the potential in the EPD process is linear, the most important dependencies of a given material on the parameters of its formation are nonlinear (e.g. specific gravity from $\mathrm{RuO}_{2}$ amount, specific capacity from weight of the electrode). Herewith, exist an optimal value for parameters of process and suspension consumption, about $100 \mathrm{~V}$ of potential and $5 \mathrm{mg} \mathrm{RuO}_{2}$ in suspension, lead to optimal usage of material and maximum capacity of electrode. This dependence is generally true for both one deposition cycle and five, while the efficiency of material use decreases with an increase in the number of cycles. This fact shows that with an increase in the layer thickness, more and more of it is not used in the process of charge accumulation, which may be associated with the compaction of the material and a decrease in the porosity of the electrode. 
Funding: The work is supported by the Ministry of Science and Higher Education of the Russian Federation (project No. 0591-2021-0002) and Russian Federation President's grant (MK-1823.2021.4).

Conflicts of Interest: The authors declare no conflict of interests.

\section{References}

1- Dr. N. R. Chodankar, Prof. Y.-K. Han / Small 2020, 2002806

2 - T. Brousse, D. Belanger, J. W. Long / J. Electrochem. Soc. 2015, 162, A5185.

3- Seung-Chul Hong, Sanghoon Kim, Woo-Jin Jang er al/ RSC Adv., 2014, 4, 48276

4 - B. J. Lee, S. Sivakkumar, J. M. Ko, J. Power Sources., 2007, 168, 546

5 - B.E. Conway, V. Birss, J. Wojtowicz, J. Power Sources., 1997, 66, 1

6 - H. Kim, B. N. Popov, J. Power Sources., 2002, 104, 52-61

7 - J. H. Jang, A. Kato, K. Machida, and K. Naoi, J. Electrochem. Soc., 2006, 153, A321- A328

8- J. P. Zheng, Solid. State. Lett., 1999, 2, 359-361

9- X. Zhang, W. Shi, J. Zhu, W. Zhao, J. Ma, S. Mhaisalkar, T. L. Maria, Y. Yang, H.

10 - Zhang and H. H. Hng, Nano Res., 2010, 3, 643

11 - A. Kumar, S. Ramaprabhu, J. Phys. Chem. C., 2011, 115, 14006-14013

12 - J. Zhang, J. Jiang, Energy Environ. Sci., 2011, 4, 4009-4015

13 - Y. F. Su, F. Wu, L. Y. Bao, New Carbon Mater., 2007, 22(1), 53-57

14 - Y. Liu, B. Zhang,Q. Xu et al. // Advanced Functional Materials. - 2018. - Vol. 28. - №21. - P. 1706592-1-1706592-12

15 - B.D. Boruah, A. Maji, A. Misra // ACS Appl. Mater. Interfaces. - 2018. - Vol. 10. - №18. - P. 15864-15872

16 - Xingyan Wang, Xianyou Wang, W. Huang et al. // Journal of Power Sources. - 2005. - Vol. 140. - №1. - P. 211-215

17 - D. Qi, Z. Liu,Y. Liu et al. // Advanced Materials. - 2015. - Vol. 27. - №37. - P. 5559-5566

18 - X. Mao, J. Xu, X. He et al. // Applied Surface Science - 2018. - Vol. 435. - P. 1228-1236

19 - X. Mao, J. Xu, X. He et al. // Applied Surface Science - 2018. - Vol. 435. - P. 1228-1236

20 - A. Alekseyev, E. Lebedev, D. Gromov et al. // PROCEEDINGS OF THE 2019 IEEE CONFERENCE OF RUSSIAN YOUNG RESEARCHERS IN ELECTRICAL AND ELECTRONIC ENGINEERING (EICONRUS). - 2019. - Vol. 1. - P. 1965-1969

21 - I. H. Kim, K. B. Kim, J. Electrochem. Soc., 2006, 153, A383-389 